\title{
Relationship of Gait and Cognition in the Elderly
}

\author{
Raminder Parihar • Jeannette R. Mahoney • Joe Verghese
}

Published online: 16 June 2013

(C) Springer Science+Business Media New York 2013

\begin{abstract}
Gait and cognitive impairments in older adults mostly reflect the co-occurrence of two geriatric syndromes linked by common underlying brain substrates and pathologies. Gait control is predominately mediated by frontal subcortical circuits, which overlap with circuits controlling executive control and attention functions. These circuits are vulnerable to multiple age-related pathologies such as ischemia, inflammation, and neurodegeneration, which could ultimately cause cognitive, gait, or combined cognitive and gait impairments. The following review aims to describe various gait and cognitive classifications, gait based phenotypes, common underlying pathological processes, and provide a link between motor and cognitive impairments in an effort to predict the risk of dementia and remediate impairments by applying appropriate interventions.
\end{abstract}

R. Parihar J. R. Mahoney $\cdot$ J. Verghese

Department of Neurology, Albert Einstein College of Medicine,

Bronx, NY, USA

R. Parihar

e-mail: raminder.parihar@gmail.com

J. R. Mahoney

e-mail: jeannette.mahoney@einstein.yu.edu

J. Verghese

Department of Medicine, Albert Einstein College of Medicine,

Bronx, NY, USA

\section{J. Verghese $(\varangle)$}

Division of Cognitive \& Motor Aging, Saul R Korey Department of

Neurology, Albert Einstein College of Medicine, 1165 Morris Park

Avenue, Rousso 301, Bronx, NY 10461, USA

e-mail: joe.verghese@einstein.yu.edu

R. Parihar

Saul R Korey Department of Neurology, Albert Einstein College of Medicine, 1165 Morris Park Avenue, Rousso 301, Bronx, NY

10461, USA

\section{J. R. Mahoney}

Division of Cognitive \& Motor Aging, Saul R Korey Department of Neurology, Albert Einstein College of Medicine, 1165 Morris Park Avenue, Rousso 304, Bronx, NY 10461, USA
Keywords Gait · Cognition · Gaitphenotypes · Inflammation · Neurodegeneration · Vascular · Quantitative gait measurements · Dual-task $\cdot$ Dementia risk $\cdot$ Remediation

\section{Introduction}

According to the U.S. Census projections, the current population of adults age 65 and older will more than double between now and 2060, from 43.1 to 92.0 million [1]. This growing aging segment of the population has brought added urgency to understanding the normal aging process, as well as age related diseases. Typically, the 'normal aging' process entails a myriad of alterations in sensory, motor, and cognitive functions that have been linked to poor quality of life [2-4], functional decline [5], increased risks of falls [6-9], and impaired mobility [5]. Additionally, disease-related motor impairments, including gait disorders and slowing of movements, are increasingly common with advancing age. A population-based study in the U.S. showed a $35 \%$ prevalence of clinically diagnosed gait disorders among communitydwelling persons over age 70 [10]. The prevalence of cognitive impairments of varying severity also increases with age, with one study reporting that $17 \%$ of adults age 65 years and older in their population-based cohort had cognitive impairments without meeting criteria for dementia and $8 \%$ had frank dementia [11]. Increasingly, the co-existence of both gait and cognitive impairments in aging has also been recognized. For instance, Camicoli and colleagues reported that gait and postural impairments were seen in 25 to $30 \%$ of cognitively normal participants and in 46 to $53 \%$ of cognitively impaired participants in the Canadian Study of Health and Aging [12].

While the co-occurrence of gait and cognitive impairments in older adults may reflect a simple co-existence of common age-related syndromes [12], others have proposed that the co-occurrence of these two geriatric syndromes may be related to a common underlying pathology $[13,14]$. Intact gait control requires the efficient integration of many neural systems, including motor, sensory, and cognitive processes, and cognitive sub-systems such as memory, attention, and 
executive function $[13,15]$. Gait control is predominately mediated by frontal subcortical circuits; circuitry which is also known to mediate executive control and attention functions $[16,17]$. Furthermore, the 'normal aging' process renders this circuitry vulnerable to multiple pathologies, such as ischemia, inflammation, and neurodegeneration, which in turn could potentially trigger cognitive, motor, or combined cognitive and motor impairments in older patients [18-20].

In the following sections, we review gait and cognitive classifications, common pathological processes for cognitive and motor syndromes, clinical utility of diagnosing combined gait and cognitive impairments, and discuss emerging intervention strategies that build on the interplay between gait and cognitive functions.

\section{Phenotyping Gait and Cognition}

\section{Clinical Gait}

Gait impairments can be classified as either neurological or non-neurological subtypes following clinical examination of walking patterns. Neurological gait abnormalities result from focal or diffuse lesions affecting the neural pathways that link cortical motor centers to the peripheral neuromuscular systems [21]. Neurological gait abnormalities are further classified into: unsteady, ataxic, neuropathic, frontal, Parkinsonian, hemiparetic and spastic subtypes [21, 22]. Nonneurological gait abnormalities result from physical limitations to walking, such as arthritis or foot deformities and do not involve the neuroaxis. Combinations of neurological or non-neurological subtypes also exist in older adults.

An alternate clinical gait classification divides gait abnormalities into low, middle and high level disorders [23, 24]. In low level gait disorders, only one major afferent sensory system (visual, proprioceptive, or vestibular) is affected. Middle level disorders result from spasticity (due to myelopathy from cervical spondylosis and stroke), Parkinsonism, or cerebellar ataxia. High level gait disorders include cautious gait, frontal gait disorders, and psychogenic gait disorders, and are thought to result from disruptions in cortico-cortical and cortico-subcortical connections.

The reliability and validity of most descriptive gait classification systems have not been verified and many gait subtypes overlap. For instance, the terms marche a petit pas, cautious gait, and apractic gait are used to describe gait abnormalities that share clinical features such as slowness, short steps, or wide base with frontal gaits. This lack of consistency may in part explain why there is a paucity of studies that have employed clinical gait subtypes, an essential part of the neurological evaluation, to predict geriatric outcomes.

Accurate identification of neurological gait sub-types enables anatomical localization of lesions, guides investigations, and provides hints to the underlying pathology. Identifying gait subtypes is also helpful in risk prognostication for motor and cognitive outcomes in clinical practice. For instance, neuropathic gaits that present with foot drops were reported to be associated with increased risk for falls [25], whereas frontal gait disorders that present with short shuffling steps with difficulty lifting the feet off the ground were reported to be associated with increased risk of developing dementia, especially vascular dementia [21].

\section{Cognitive Syndromes}

Cognitive disorders, unlike gait disorders, are categorized on a spectrum of cognitive decline, beginning with cognitive normalcy, transitioning to intermediate states such as the mild cognitive impairment (MCI), and often reaching an endpoint of dementia. MCI is defined as an impairment in one or more domains of cognitive function, without interference in daily activities in non-demented individuals [26]. It is further sub-classified into three categories: one that predominately involves memory impairments (amnestic MCI); a second that involves impairments in cognitive domains other than memory (non-amnestic MCI), such as executive function, language, or visuospatial impairments; and a third that involves multiple impairments across both memory and other cognitive domains (combined MCI) [27]. Patients who meet MCI criteria in clinical practice are at higher risk of transitioning to dementia [28].

Presence of neurological gait subtypes as well as quantitative gait impairments have been linked to $\mathrm{MCI}[21,29,30]$. Recent studies suggest that gait slowing may precede declines in cognitive tests in older adults [31, 32•]. Hence, gait may complement cognitive assessments in MCI. The role of gait assessment in predicting transitions to dementia in MCI patients requires further investigation.

\section{Quantitative Gait}

When gait impairments are subtle, they may not be detected by the untrained eye. Quantitative measures provide an objective means of assessing gait that minimizes examiner bias. In our studies, a computerized walkway with embedded pressure sensors (GAITRite, CIR systems) is used to measure gait $[29,33,34]$. Participants are asked to walk on the mat at their 'normal pace' in a quiet well-lit hallway wearing comfortable footwear and without any attached monitoring devices. Based on footfalls recorded on the walkway, the software automatically computes numerous gait variables. We employ eight parameters to describe gait performance [33-35]: velocity $(\mathrm{cm} / \mathrm{sec})$; cadence (steps/minute); stride length $(\mathrm{cm})$; swing time (sec); stance time (sec); and double support phase (\%); as well as standard deviation (SD) of stride length and SD of swing time, both of which serve as 
proxies for gait variability [29]. Gait variability has been linked to increased risk of falls and dementia in older adults $[34,35]$.

These eight gait variables have been subjected to factor analysis to derive gait domains of pace, rhythm, and variability $[34,35]$. This statistical approach accounts for colinearity between individual gait variables, as well as affords an opportunity to assess specific gait domains. In our and other studies [34, 35], the pace factor was accounted for by velocity and stride length, while the rhythm factor was accounted for by cadence and timing, and the variability factor by gait variability in stride length and swing time. While the pace domain predicted decline in executive function, rhythm predicted decline in memory, and gait variability predicted risk of dementia [34]. Thus, particular facets of gait were linked to specific cognitive domains, raising the possibility of a shared biology between circuitry.

Early motor dysfunction, assessed by presence of gait slowing, co-exists with or even precedes the onset of cognitive decline in older adults [36]. It is assumed that stride length and velocity, factors that load on pace, are controlled by phasic output from the basal ganglia to the supplementary motor area, whereas spinal and brainstem pathways determine cadence (rhythm) [37]. Parkinsonian gait has been correlated with presence and severity of substantia nigra neurofibrillary tangles, even in brain autopsy studies of individuals without clinical Alzheimer's disease or with minimal Alzheimer pathology [38]. Therefore, it is possible that Alzheimer pathology may involve gait regions in the brain very early on in its course, and quantitative gait assessments could provide early diagnostic clues.

\section{Linking Gait and Cognition}

\section{Attention and Gait}

Converging evidence from clinical, neuroscience, and neuroimaging studies indicate a major role for executive attention processes and frontal-subcortical circuits in mobility in older adults $[13,14,18,39,40]$. Increased levels of attention are associated with better gait performance [15, 41-43]. This association is best demonstrated using dual-task methodology, which simultaneously assesses cognitive and motor task performance by requiring individuals to walk while performing a cognitively demanding task. For instance, the 'Walking While Talking' test is a cognitive-motor divided attention task that requires individuals to walk while reciting alternate letters of the alphabet. The goal of this test is to unmask latent mobility abnormalities by increasing the complexity of the walking condition [43-46]. In this paradigm, gait parameters such as velocity and stride length in the dual-task condition (walking while talking) can be directly compared to gait parameters in the single-task condition (walking without talking) [47]. Changes in gait performance during the dual-task (also called 'dual task cost') support the functional role of attention during walking. Our work indicates that cognitive domains such as executive attention and memory are associated with velocity during normal pace walking as and walking while talking; supporting the notion that walking is a complex task requiring the involvement of higher-order cortical control processes [13].

The clinical utility of dual-task conditions has been reported; gait speed during walking while talking is a strong predictor of risk of falls [45]. The walking while talking test might also serve as a mobility stress test by increasing the cognitive demands of walking. For instance, the walking while talking test predicted future risk of developing frailty, disability and death in high functioning non-disabled older adults with normal walking speeds [48].

\section{Motor Cognitive Risk Syndrome}

Despite growing evidence of the link between cognition and motor performance in aging, there have been limited attempts to capitalize on these findings in dementia risk prognostications. The recently described Motoric Cognitive Risk (MCR) syndrome attempts to identify individuals who are at high-risk for developing dementia [49•]. A patient meets criteria for the MCR syndrome if he/she demonstrates 1) cognitive complaints; 2) slow gait (velocity one SD or more below age and sex appropriate mean values); 3) preserved activities of daily living (ADL); and 4) absence of dementia. Thus, the MCR criteria are similar to those employed to define MCI, with the exception of the objective cognitive criteria in MCI being substituted by the slow gait requirement in MCR syndrome. MCR has strong predictive validity for dementia: older participants meeting criteria for MCR were over three times likely to develop dementia and more than twelve times likely to develop vascular dementia. Interestingly, MCR syndrome was a better predictor of dementia than cognitive complaints or slow gait alone. While there is overlap between MCR and MCI cases, MCR syndrome still predicted risk of dementia after accounting for MCI subtypes.

\section{Gait and Cognitive Disorders Share Common Pathology}

Herein, we describe a few selected pathological processes that are common in aging and are associated with decline in cognitive and motor functions. A better understanding of the common pathological processes that contribute to decline in gait and cognitive functions can be used as a springboard to develop new interventions to prevent or diminish gait and cognitive impairments in the elderly. 


\section{Vascular}

Vascular risk factors such as hypertension can lead to cerebral ischemia secondary to impairment in arterial vasoreactivity, obstruction of small subcortical arterioles, or hypoperfusion [18]. Vascular lesions can be either focal, resulting in lacunar infarctions, seen in the thalamus, basal ganglia, internal capsule or brainstem [50], or diffuse, affecting the periventricular white matter [51]. The periventricular white matter consists of the ascending thalamocortical and descending corticospinal tracts, which sub-serve gait and balance functions [16]. The frontal-subcortical connections, which control speed of cognitive processing and executive function, also travel within the periventricular white matter [17]. Due to the close proximity of these two circuits, white matter lesions (WML) may affect motor and cognitive functions simultaneously. In elderly patients, WML are strongly associated with cardiovascular disease such as hypertension, diabetes, or hyperlipidemia [52]. Research has shown that accumulation of vascular structural abnormalities, in both white and gray matter accounts for cognitive decline [53-55] and gait impairment [14, 56, 57]. The severity of periventricular WML is associated with a decline in the speed of mental processing [54], as well as lower performance in fluid intelligence measures $[53,55]$. Subclinical white matter hyperintensities, brain infarcts and brain atrophy predicted a faster rate of decline in gait speed over time [56]. In addition to velocity, Rosano and colleagues demonstrated that a decline in stride length, and an increase in step length variability, was indicative of the presence of brain infarcts and white matter hyperintensities in an elderly population free from stroke, dementia, or other neurological diseases [14, 57].

\section{Inflammation}

While normal aging is associated with a low-grade systemic inflammation, previous studies have linked higher levels of inflammatory markers to impairments in mobility and physical function in older adults [19, 58, 59]. Cross-sectional studies have demonstrated an association between high serum levels of inerleukin-6 (IL-6) and tumor necrosis factor alpha (TNF- $\alpha$ ) with worsening of functional and mobility status [60-62]. However, only elevated serum levels of IL-6 but not TNF- $\alpha$ were associated with increased rates of decline in gait speed when examined prospectively [63]; suggesting that not all inflammatory markers are involved in motoric decline. Inflammation is also implicated in the cascade that leads to the development of amyloid neuritic plaques, one of the pathological hallmarks of Alzheimer's disease (AD) [64]. Epidemiological studies have identified associations between specific inflammatory markers, such as increased IL-6 levels with a decline in the ability to encode new information and recall learned information; aspects of cognition affected early in $\mathrm{AD}$ [65].
Thus, declines in gait speed and memory impairment share a common increase in the same inflammatory markers. Reduction or prevention of inflammation could be explored as a possible new avenue to slow of or halt the progression of cognitive and gait decline in aging [66].

\section{Neurodegeneration}

Neurodegenerative diseases, like Parkinson's disease, also demonstrate the intersection between cognition and motor impairment in that specific facets of cognition have been related to different motor phenotypes [20]. Bradykinesia is associated with reduced mental flexibility and decreased working memory abilities [20]. Presence of postural instability and gait disturbances is associated with impaired visuospatial function and memory [20]. Hence, cognitive impairment co-exists with motor decline even in the early stages of neurodegenerative diseases such as Parkinson's disease. Imaging studies further support this notion. When dopamine activity was measured in patients with impaired executive function, particularly, impaired working memory, there was a decrease in dopamine activation in both the basal ganglia and/or frontal cortex [67, 68]. Such results signify that diseases resulting from diminished dopamine, not only affect motor abilities, due to basal ganglia involvement, but they also affect cognition, due to involvement of the frontal circuits responsible for executive function.

\section{Interventions}

\section{Physical Activity and Dementia Risk}

Physical activity contributes to healthy aging and reduces morbidity and mortality [69]. Physical activity has been shown to have protective effects against mortality in patients with chronic diseases such as cardiovascular disease [70]. Many studies have examined the effect of physical activity on cognitive performance in older individuals. For instance, Baker and colleagues demonstrated an improvement in executive function tests in individuals with normal cognition who underwent a 6-month aerobic exercise program compared to healthy controls who participated in a stretching regimen [71]. The researchers further demonstrated an improvement in executive function after a 6-month aerobic exercise program in individuals with MCI [72]. A French study enrolled patients with dementia from a nursing home, half of whom took part in three 60-minute exercise sessions a week that strategically focused on improving walking, stamina and equilibrium [73]. The 31 individuals who underwent the physical intervention showed improvement in composite cognitive functions, while the 16 participants in the usual care control group showed a decline in cognitive functions. 
Collectively, these studies support the link between gait and cognition in that physical exercise results in improvements in cognition.

\section{Cognitive Remediation and Mobility}

The reverse relationship between cognitive interventions and their effect of gait are being explored in recent studies. Cognitive remediation approaches using computerized programs or cognitive training have demonstrated an improvement in attention and executive function as well as memory in cognitively normal older adults. Verghese and colleagues [74•] conducted a pilot study in which 24 frail older adults were randomly assigned to either participate in a computerized cognitive remediation program or were in a usual care group for a 12-week period. The cognitive remediation group showed an improvement in gait velocity during normal walking and during walking while talking conditions compared to their baseline performance. This small study suggests the possibility that cognitive remediation could be a new, non-pharmacological means of modifying gait performance, especially during dual-task conditions.

Participants who received training in dual-tasking have demonstrated improvements in walking abilities $[75,76]$. Schwenk and colleagues evaluated the efficacy of a 12-week dual-task training program in seniors with dementia. The participants were randomized to either a dual-task exercise session, involving walking while performing complex motor or cognitive activities, or a low intensity exercise session [75]. After 12 weeks, the group that received dual-task training performed significantly better on gait in a complex dual- task condition compared to the control group. Mirelman and colleagues examined the efficacy of a treadmill training program enhanced with virtual reality in patients with Parkinson's disease [76]. After six weeks of training, gait velocity, stride time and stride length significantly improved in normal and dual-tasking conditions, and gait variability decreased (improved) under the dual-task condition.

These early studies support the feasibility and validity of cognitive based approaches to improve mobility. The effect of these interventions seems especially beneficial in cognitively demanding dual-task walking conditions. Larger well-designed studies are needed to further validate this new approach.

\section{Conclusions}

Review of the literature suggests that the co-existence of gait and cognitive impairments in older adults is related to a common underlying pathology and is not an age-related phenomenon. Gait control is predominately mediated by frontal subcortical circuits, which overlap with circuits controlling executive control and attention functions. Therefore, any pathological process affecting one circuit could affect the other resulting in combined cognitive and motor impairments in older patients. Clinical and quantitative gait assessments, as well as a clinical gait based phenotypes such as the MCR syndrome, could serve as risk prognosticators in clinical and research settings to predict various geriatric syndromes such as falls, frailty, disability, dementia, and death. Emerging evidence from pilot studies also highlights the potential role of cognitive and motor remediation interventions to prevent further decline in either gait or cognitive function. Further studies are needed to understand the common biology and brain substrates underlying cognitive-motor impairments in aging as a prelude to improving current risk assessment procedures and developing novel interventions to maintain functional independence in older individuals.

\section{Compliance with Ethics Guidelines}

Conflict of Interest Raminder Parihar declares that she has no conflict of interest.

Jeannette R. Mahoney declares that she has no conflict of interest. Joe Verghese is supported by a grant from the National Institutes of Health (NIH).

Human and Animal Rights and Informed Consent This article does not contain any studies with human or animal subjects performed by any of the authors.

\section{References}

Papers of particular interest, published recently, have been highlighted as:

- Of importance

1. U.S. Census Bureau Projections show a slower growing, older, more diverse nation a half century from now. Available at https://www.census.gov/newsroom/releases/archives/population/ cb12-243.html. Accessed April 2013.

2. Appollonio I, Carabellese C, Frattola L, Trabucchi M. Effects of sensory aids on the quality of life and mortality of elderly people: a multivariate analysis. Age Ageing. 1996;25(2):89-96.

3. Carabellese C, Appollonio I, Rozzini R, et al. Sensory impairment and quality of life in a community elderly population. J Am Geriatr Soc. 1993;41(4):401-7.

4. Yueh B, Shapiro N, MacLean CH, Shekelle PG. Screening and management of adult hearing loss in primary care: scientific review. JAMA. 2003;289(15):1976-85.

5. Kaye JA, Oken BS, Howieson DB, et al. Neurologic evaluation of the optimally healthy oldest old. Arch Neurol. 1994;51(12):1205-11.

6. Camicioli R, Panzer VP, Kaye J. Balance in the healthy elderly: posturography and clinical assessment. Arch Neurol. 1997;54(8):976-81.

7. Judge JO, King MB, Whipple R, et al. Dynamic balance in older persons: effects of reduced visual and proprioceptive input. J Gerontol A Biol Sci Med Sci. 1995;50(5):M263-70. 
8. Lord SR, Ward JA, Williams P, Anstey KJ. Physiological factors associated with falls in older community-dwelling women. J Am Geriatr Soc. 1994;42(10):1110-7.

9. Lord SR, Rogers MW, Howland A, Fitzpatrick R. Lateral stability, sensorimotor function and falls in older people. J Am Geriatr Soc. 1999;47(9): 1077-81.

10. Verghese J, LeValley A, Hall CB, et al. Epidemiology of gait disorders in community-residing older adults. J Am Geriatr Soc. 2006;54(2):255-61.

11. Graham JE, Rockwood K, Beattie BL, et al. Prevalence and severity of cognitive impairment with and without dementia in an elderly population. Lancet. 1997;349(9068):1793-6.

12. Camicioli R, Wang Y, Powell C, et al. Gait and posture impairment, parkinsonism and cognitive decline in older people. J Neural Transm. 2007;114(10):1355-61

13. Holtzer R, Verghese J, Xue X, Lipton RB. Cognitive processes related to gait velocity: results from the Einstein Aging Study. Neuropsychology. 2006;20(2):215-23.

14. Rosano C, Brach J, Longstreth Jr WT, Newman AB. Quantitative measures of gait characteristics indicate prevalence of underlying subclinical structural brain abnormalities in high-functioning older adults. Neuroepidemiology. 2006;26(1):52-60.

15. Scherder E, Eggermont L, Swaab D, et al. Gait in ageing and associated dementias; its relationship with cognition. Neurosci Biobehav Rev. 2007;31(4):485-97.

16. Thompson PD. Gait disorders accompanying diseases of the frontal lobes. Adv Neurol. 2001;87:235-41.

17. Alvarez JA, Emory E. Executive function and the frontal lobes: a meta-analytic review. Neuropsychol Rev. 2006;16(1):17-42.

18. Pugh KG, Lipsitz LA. The microvascular frontal-subcortical syndrome of aging. Neurobiol Aging. 2002;23(3):421-31.

19. Maggio M, Guralnik JM, Longo DL, Ferrucci L. Interleukin-6 in aging and chronic disease: a magnificent pathway. J Gerontol A Biol Sci Med Sci. 2006;61(6):575-84.

20. Domellof ME, Elgh E, Forsgren L. The relation between cognition and motor dysfunction in drug-naive newly diagnosed patients with Parkinson's disease. Mov Disord. 2011;26(12):2183-9.

21. Verghese J, Lipton RB, Hall CB, et al. Abnormality of gait as a predictor of non-Alzheimer's dementia. N Engl J Med. 2002;347(22):1761-8.

22. Sudarsky L. Geriatrics: gait disorders in the elderly. N Engl J Med. 1990;322(20):1441-6.

23. Nutt JG, Marsden CD, Thompson PD. Human walking and higherlevel gait disorders, particularly in the elderly. Neurology. 1993;43(2):268-79.

24. Nutt JG. Classification of gait and balance disorders. Adv Neurol. 2001;87:135-41.

25. Verghese J, Ambrose AF, Lipton RB, Wang C. Neurological gait abnormalities and risk of falls in older adults. J Neurol. 2010;257(3):392-8.

26. Petersen RC, Negash S. Mild cognitive impairment: an overview. CNS Spectr. 2008;13(1):45-53.

27. Petersen RC. Mild cognitive impairment as a diagnostic entity. $J$ Intern Med. 2004;256(3):183-94.

28. Petersen RC. Clinical practice. Mild cognitive impairment. N Engl J Med. 2011;364(23):2227-34.

29. Verghese J, Robbins M, Holtzer R, et al. Gait dysfunction in mild cognitive impairment syndromes. J Am Geriatr Soc. 2008;56(7):1244-51.

30. McGough EL, Kelly VE, Logsdon RG, et al. Associations between physical performance and executive function in older adults with mild cognitive impairment: gait speed and the timed "up \& go" test. Phys Ther. 2011;91(8):1198-207.

31. Mielke MM, Roberts RO, Savica R, et al. Assessing the temporal relationship between cognition and gait. Slow gait predicts cognitive decline in the Mayo Clinic Study of Aging. J Gerontol A Biol Sci Med Sci. 2012; In press.

32. - Buracchio T, Dodge HH, Howieson D, et al. The trajectory of gait speed preceding mild cognitive impairment. Arch Neurol. 2010;67(8):980-6.
The investigators demonstrated that gait slowing precedes decline on cognitive tests by many years in older adults with mild cognitive impairment syndrome.

33. Abellan van Kan G, Rolland Y, Andrieu S, et al. Gait speed at usual pace as a predictor of adverse outcomes in community-dwelling older people an International Academy on Nutrition and Aging (IANA) Task Force. J Nutr Health Aging. 2009;13(10):881-9.

34. Verghese J, Wang C, Lipton RB, et al. Quantitative gait dysfunction and risk of cognitive decline and dementia. J Neurol Neurosurg Psychiatry. 2007;78(9):929-35.

35. Verghese J, Holtzer R, Lipton RB, Wang C. Quantitative gait markers and incident fall risk in older adults. J Gerontol A Biol Sci Med Sci. 2009;64(8):896-901.

36. Camicioli R, Howieson D, Oken B, et al. Motor slowing precedes cognitive impairment in the oldest old. Neurology. 1998;50(5):1496-8.

37. Drew T, Prentice S, Schepens B. Cortical and brainstem control of locomotion. Prog Brain Res. 2004;143:251-61.

38. Schneider JA, Li JL, Li Y, et al. Substantia nigra tangles are related to gait impairment in older persons. Ann Neurol. 2006;59(1):166-73.

39. Rosano C, Aizenstein HJ, Studenski S, Newman AB. A regions-ofinterest volumetric analysis of mobility limitations in communitydwelling older adults. J Gerontol Biol Med Sci. 2007;62(9):1048-55.

40. Holtzer R, Friedman R, Lipton RB, et al. The relationship between specific cognitive functions and falls in aging. Neuropsychology. 2007;21(5):540-8.

41. Chen HC, Schultz AB, Ashton-Miller JA, et al. Stepping over obstacles: dividing attention impairs performance of old more than young adults. J Gerontol Biol Sci Med Sci. 1996;51(3):M116-22.

42. Barberger-Gateau P, Fabrigoule C. Disability and cognitive impairment in the elderly. Disabil Rehabil. 1997;19(5):175-93.

43. Sparrow WA, Bradshaw EJ, Lamoureux E, Tirosh O. Ageing effects on the attention demands of walking. Hum Mov Sci. 2002;21(56):961-72.

44. Camicioli R, Howieson D, Lehman S, Kaye J. Talking while walking: the effect of a dual task in aging and Alzheimer's disease. Neurology. 1997;48(4):955-8.

45. Verghese J, Buschke H, Viola L, et al. Validity of divided attention tasks in predicting falls in older individuals: a preliminary study. J Am Geriatr Soc. 2002;50(9):1572-6.

46. Li KZ, Lindenberger U, Freund AM, Baltes PB. Walking while memorizing: age-related differences in compensatory behavior. Psychol Sci. 2001;12(3):230-7.

47. Woollacott M, Shumway-Cook A. Attention and the control of posture and gait: a review of an emerging area of research. Gait Posture. 2002;16(1):1-14.

48. Verghese J, Holtzer R, Lipton RB, Wang C. Mobility stress test approach to predicting frailty, disability, and mortality in highfunctioning older adults. J Am Geriatr Soc. 2012;60(10):1901-5.

49. • Verghese J, Wang C, Lipton RB, Holtzer R. Motoric cognitive risk syndrome and the risk of dementia. J Gerontol A Biol Sci Med Sci. 2012;68(4):412-8. A cognitive-motor syndrome combining cognitive complaints with slow gait was a strong predictor of vascular dementia.

50. Fisher CM. Lacunes: small, deep cerebral infarcts. Neurology. 1965;15:774-84.

51. Cummings JL. Anatomic and behavioral aspects of frontalsubcortical circuits. Ann N Y Acad Sci. 1995;769:1-13.

52. Pantoni L, Garcia JH. Pathogenesis of leukoaraiosis: a review. Stroke. 1997;28(3):652-9.

53. Garde E, Mortensen EL, Krabbe K, et al. Relation between agerelated decline in intelligence and cerebral white-matter hyperintensities in healthy octogenarians: a longitudinal study. Lancet. 2000;356(9230):628-34.

54. de Groot JC, de Leeuw FE, Oudkerk M, et al. Cerebral white matter lesions and cognitive function: the Rotterdam Scan Study. Ann Neurol. 2000;47(2):145-51. 
55. Leaper SA, Murray AD, Lemmon HA, et al. Neuropsychologic correlates of brain white matter lesions depicted on MR images: 1921 Aberdeen Birth Cohort. Radiology. 2001;221(1):51-5.

56. Rosano C, Kuller LH, Chung H, et al. Subclinical brain magnetic resonance imaging abnormalities predict physical functional decline in high-functioning older adults. J Am Geriatr Soc. 2005;53(4):649-54.

57. Rosano C, Brach J, Studenski S, et al. Gait variability is associated with subclinical brain vascular abnormalities in high-functioning older adults. Neuroepidemiology. 2007;29(3-4):193-200.

58. Bruunsgaard $H$, Pedersen $M$, Pedersen BK. Aging and proinflammatory cytokines. Curr Opin Hematol. 2001;8(3):131-6.

59. Starr ME, Evers BM, Saito H. Age-associated increase in cytokine production during systemic inflammation: adipose tissue as a major source of IL-6. J Gerontol A Biol Sci Med Sci. 2009;64(7):723-30.

60. Penninx BW, Kritchevsky SB, Newman AB, et al. Inflammatory markers and incident mobility limitation in the elderly. J Am Geriatr Soc. 2004;52(7):1105-13.

61. Taaffe DR, Harris TB, Ferrucci L, et al. Cross-sectional and prospective relationships of interleukin- 6 and C-reactive protein with physical performance in elderly persons: MacArthur studies of successful aging. J Gerontol A Biol Sci Med Sci. 2000;55(12):M709-15.

62. Cesari M, Penninx BW, Pahor M, et al. Inflammatory markers and physical performance in older persons: the InCHIANTI study. J Gerontol A Biol Sci Med Sci. 2004;59(3):242-8.

63. Verghese J, Holtzer R, Oh-Park M, et al. Inflammatory markers and gait speed decline in older adults. J Gerontol A Biol Sci Med Sci. 2011;66(10):1083-9.

64. McGeer PL, McGeer EG. Inflammation, autotoxicity and Alzheimer disease. Neurobiol Aging. 2001;22(6):799-809.

65. Elderkin-Thompson V, Irwin MR, Hellemann G, Kumar A. Interleukin-6 and memory functions of encoding and recall in healthy and depressed elderly adults. Am J Geriatr Psychiatr. 2012;20(9):753-63.

66. Launer LJ. Nonsteroidal anti-inflammatory drugs and Alzheimer disease: what's next? JAMA. 2003;289(21):2865-7.
67. Lewis SJ, Dove A, Robbins TW, et al. Cognitive impairments in early Parkinson's disease are accompanied by reductions in activity in frontostriatal neural circuitry. J Neurosci. 2003;23(15):6351-6.

68. Marklund P, Larsson A, Elgh E, et al. Temporal dynamics of basal ganglia under-recruitment in Parkinson's disease: transient caudate abnormalities during updating of working memory. Brain. 2009;132(Pt 2):336-46.

69. Byberg L, Melhus H, Gedeborg R, et al. Total mortality after changes in leisure time physical activity in 50-year-old men: 35 year follow-up of population based cohort. BMJ. 2009;338:b688.

70. Kodama S, Saito K, Tanaka S, et al. Cardiorespiratory fitness as a quantitative predictor of all-cause mortality and cardiovascular events in healthy men and women: a meta-analysis. JAMA. 2009;301(19):2024-35.

71. Baker LD, Frank LL, Foster-Schubert K, et al. Aerobic exercise improves cognition for older adults with glucose intolerance, a risk factor for Alzheimer's disease. J Alzheimers Dis. 2010;22(2):569-79.

72. Baker LD, Frank LL, Foster-Schubert K, et al. Effects of aerobic exercise on mild cognitive impairment: a controlled trial. Arch Neurol. 2010;67(1):71-9.

73. Kemoun G, Thibaud M, Roumagne N, et al. Effects of a physical training program on cognitive function and walking efficiency in elderly persons with dementia. Dement Geriatr Cogn Disord. 2010;29(2):109-14.

74. - Verghese J, Mahoney J, Ambrose AF, et al. Effect of cognitive remediation on gait in sedentary seniors. J Gerontol A Biol Sci Med Sci. 2010;65(12):1338-43. This study demonstrates that mobility may be improved by intervening on underlying cognitive processes and suggests a new intervention strategy.

75. Schwenk M, Zieschang T, Oster P, Hauer K. Dual-task performances can be improved in patients with dementia: a randomized controlled trial. Neurology. 2010;74(24):1961-8.

76. Mirelman A, Maidan I, Herman T, et al. Virtual reality for gait training: can it induce motor learning to enhance complex walking and reduce fall risk in patients with Parkinson's disease? J Gerontol A Biol Sci Med Sci. 2011;66(2):234-40. 